\title{
生物大分子可视化技术综述
}

\author{
郭栋梁 ${ }^{1,2)}$, 王艳芬 ${ }^{3}$, 李煜 ${ }^{1)}$, 郭瑶瑶 1 ), 徐锡明4)，聂俊岗 ${ }^{1)}$ \\ 1) (燕山大学信息科学与工程学院 秦皇岛 066004) \\ 2) (河北省软件工程重点实验室 秦皇岛 066004) \\ 3) (燕山大学工程训练中心 秦皇岛 066004) \\ 4) (中国海洋大学医药学院 青岛 266003) \\ (wyf@ysu.edu.cn)
}

\begin{abstract}
摘 要: 生物大分子, 如蛋白质、核酸, 影响着生命体的微观行为, 直接决定了物种的生命功能. 生物大分子可视化 技术, 对分子生物学和生物化学领域具有重要的研究意义, 是计算机辅助药物设计的重要研究方向. 文中对近年来 生物大分子可视化进行深人的分析. 首先从分子结构的空间形态定义出发，概述分子骨架、表面、空腔 3 种基本空间 形态的概念和可视化方法; 然后论述近年来大规模生物分子场景可视化技术，包括分子组装、多细节层次和 GPU 加 速; 再阐述动态分子可视分析技术，包括分子轨迹和空腔可视分析，进而介绍面向虚拟现实的生物分子可视化交互 技术；最后，总结生物大分子可视化研究面临的挑战，并对其未来发展趋势进行展望.
\end{abstract}

关键词：计算机辅助药物设计; 分子可视化; 可视分析; 分子组装; 分子轨迹; 分子空腔

中图法分类号: TP391.41ＤOI: 10.3724/SP.J.1089.2021.19265

\section{Survey on Visualization of Biomacromolecule}

\author{
Guo Dongliang ${ }^{1,2)}$, Wang Yanfen ${ }^{3)^{*}}, \mathrm{Li} \mathrm{Yu}^{1)}$, Guo Yaoyao ${ }^{1)}, \mathrm{Xu} \mathrm{Ximing}^{4)}$, and Nie Junlan ${ }^{1)}$ \\ 1) (School of Information Science and Engineering, Yanshan University, Qinhuangdao 066004) \\ 2) (Key Laboratory of Software Engineering of Hebei Province, Qinhuangdao 066004) \\ 3) (Engineering Training Center, Yanshan University, Qinhuangdao 066004) \\ 4) (School of Medicine and Pharmacy, Ocean University of China, Qingdao 266003)
}

\begin{abstract}
Biomacromolecules, such as proteins and nucleic acids, affect the microscopic behavior of beings and directly determine the life functions of species. The visualization technology of biomacromolecule structure has large significance in the field of molecular biology and biochemistry, and is an important research direction of computer-aided drug design. In this paper, the visualization of biomacromolecule structure in recent years is deeply investigated and analyzed. First of all, the concept of molecular skeleton, surface and cavity and the basic visualization methods are summarized based on the definition of molecular spatial morphology. Secondly, the recent large-scale visualization technologies of biomolecule scene, including molecular assembly, LOD and GPU acceleration, are discussed. Thirdly, the visual analysis techniques of dynamic molecular structure are shown, which includes visual analysis of molecular trajectories and dynamic cavities. Then, the visual interaction technologies of biomolecules for virtual reality is introduced. Finally, the challenges faced by the visualization of biomacromolecular structures are summarized, and the future development trend is prospected.
\end{abstract}

收稿日期：2021-07-07; 修回日期：2021-10-15. 基金项目：国家自然科学基金(61802334)。郭栋梁(1983一)，男，博士，副教授， 硕士生导师, CCF 会员, 主要研究方向为数据可视化、计算机图形学; 王艳芬(1982-), 女, 硕士, 实验师, 论文通讯作者, 主要研究 方向为数据可视化; 李暗(1997一), 女, 硕士研究生, 主要研究方向为数据可视化; 郭瑶瑶(1996-), 女, 硕士研究生, 主要研究方向 为数据可视化; 徐锡明(1984-), 男, 博士, 副研究员, 硕士生导师, 主要研究方向为多学科交叉药物笁选; 聂俊岗(1962一), 女, 博 士，教授，博士生导师, CCF 会员，主要研究方向为计算机图形学、计算机仿真. 
Key words: computer-aided drug design; molecular visualization; visual analysis; molecular assembly; molecular trajectories; molecular cavity

生物分子由成千上万的原子组成，目前的测 定方法包括 $\mathrm{X}$ 射线晶体衍射分析、核磁共振波谱 分析和冷冻电镜实验. 由于生物分子是典型的空 间稠密结构, 且其结构体现重要的生物功能, 生物 化学领域的研究人员针对不同的应用场景设计了 分子的各种可视化表示形式. 这些可视化技术, 有 效地辅助了领域专家观察分析关注的结构区域, 不仅提升了科研效率，而且对科普微观生物世界 也具有重要意义.

随着生物数据测定技术的不断完善, 生物分 子结构数据量快速增长 ${ }^{[1]}$, 截至 2021 年 4 月 7 日, 已达到 176247 个结构; 随着计算机性能的不断提 升, 百万级时间步的分子动力学模拟已作为重要 的数据供科学家研究分析. 生物大分子动态可视 化的目的是快速感知和探索大规模时空生物结构 数据, 其已成为当前生物化学、生物信息学、生 物图形学与可视化等交叉领域研究的热点, 受到 广泛关注 ${ }^{[2]}$.

如图 1 所示，本文对生物分子结构定义、大规 模生物分子场景可视化及动态分子可视分析的相 关工作进行梳理，对近年生物大分子可视化技术 进行全面的调研和分析. 首先，概述了分子骨架、 表面、空腔的概念及 3 类模型的基本可视化方法; 进而论述了分子组装、多细节层次(level of detail, LOD)和 GPU 加速等大规模生物分子场景可视化 的核心技术，重点分析了分子组装和 GPU 加速渲 染的相关工作，介绍了 SARS-CoV-2 病毒结构可视

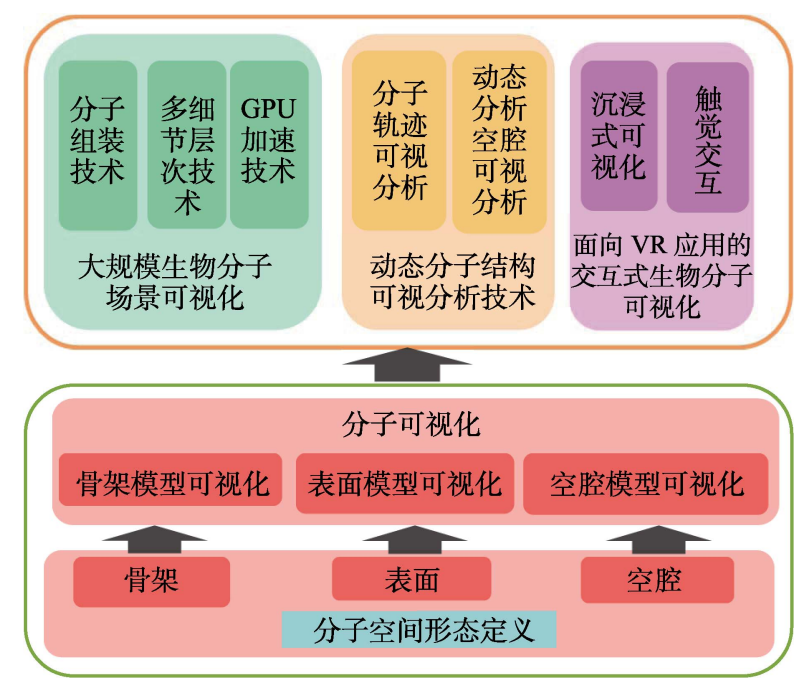

图 1 本文框架
化的工作进展; 阐述了包括分子轨迹可视分析和 空腔可视分析的动态分子可视分析技术以及生物 分子可视化交互技术; 最后, 总结了生物大分子可 视化研究面临的挑战, 展望了其未来发展的趋势.

\section{1 生物分子结构空间形态定义及可视化}

\section{1 分子结构空间形态定义}

蛋白质和核酸等生物分子由一系列特定排列 的原子组成, 其构成了密集型空间. 由于原子体积 小、空间排布紧密, 需要针对不同的感知和分析目 的设计可视化模型. 目前, 分子结构空间形态分为 骨架、表面和空腔 3 类.

分子骨架结构形态的二维示意如图 2 所示, 其 由分子内化学键按照一定的形式连接形成, 重点 描述分子中各组成部分之间的空间拓扑关系, 常 见的分子骨架模型包括描述原子间空间关系的线 模型、胶囊模型、球棒模型等, 以及从更高层次抽 象氨基酸之间二级结构关系的 Ribbon 模型和 Cartoon 模型.

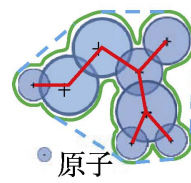

a. 分子结构

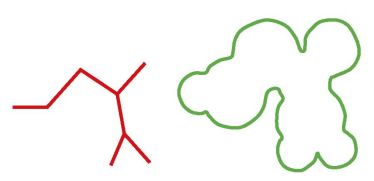

b. 骨架形态

c. 表面形态

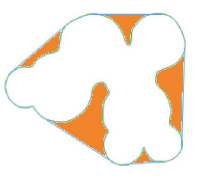

d. 空间
图 2 分子结构的空间形态结构二维示意

生物的化学和物理反应的基础是分子之间受 各种化学键和次级键的接触活动, 如图 2 所示, 定 义并描述分子表面对领域专家理解其空间结构尤 为重要. 常见的分子表面模型包括范德华球模型、 溶剂可及表面模型、溶剂排斥表面模型和 Gaussian 表面模型等.

空腔是指活性位点周围表面形成的空间, 其 大小和形状影响着分子反应的难易程度. 空腔结 构的计算模型包括基于网格、Voronoi、空腔表面 和探针等模型 ${ }^{[3]}$.

\section{2 分子骨架模型可视化}

可视化生物分子中的化学键有助于理解和预 测给定分子的许多化学性质. 分子骨架模型适合 展现分子中原子间的化学键, 是最常用的可视化 
方法之一. 由于生物化学反应通常是 2 个或多个生 物分子相互作用, 较多使用 Focus + Context 交互方 式展现该三维结构提升感知, 骨架模型被用来作 为 Context 展现用户关注的局部细节的背景三维结 构. 分子骨架模型大致分为 2 类: 第 1 类是描述原 子和原子间化学键的分子骨架模型; 第 2 类是抽象 描述氨基酸与肽键的蛋白质二级结构模型.

描述原子和原子化学键的分子可视化最简单 的代表是线模型. 更复杂的可视化用圆柱体表示 键, 用球体表示原子的球棒模型. 分子可视化工具 如可视化分子动力学工具(visual molecular dynamics, VMD $)^{[4]}$, PyMOL $^{[5]}$, Chimera ${ }^{[6]}$, MegaMol $^{[7]}$,

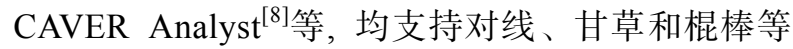
骨架模型的可视化. 如图 3a 所示, VMD 支持的原 子骨架模型有线、棒状、甘草和球棒等模型. 骨架 模型一般采用线、球和圆柱等简单几何要素, 适合 采用隐式表面描述. 近年来, 借助 GPU 光线投射技 术, 实现了较三角形网格更高的渲染效率和质量.

描述氨基酸与肽键的蛋白质二级结构，是指 将多肽主链骨架原子沿一定的轴线盘旋或折叠而 形成的特定构象，即肽链主链骨架原子的空间位 置排布, 蛋白质二级结构的形式包括 $\alpha$-螺旋、 $\beta$-折 叠、 $\beta$-转角和无规卷曲. 1981 年, Richardson ${ }^{[9]}$ 首次 提出采用管状和条带作为图形元素的 Cartoon 模型 视觉表达蛋白质二级结构. Cartoon 模型表达二级 结构的方法, 如图 3b 所示, 有管状和 Ribbon 等模 型. Cartoon 模型兼具实用性和美感，已成为最重 要的蛋白质分子展示方法之一.

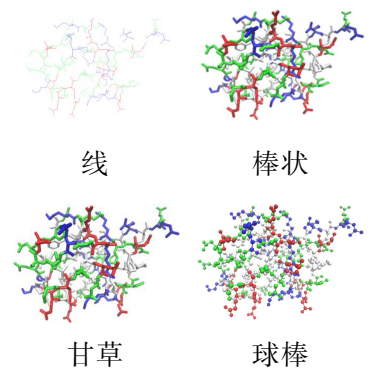

a. 原子化学键骨架模型

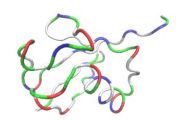

管状

Cartoon

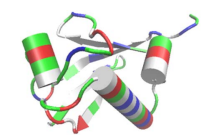

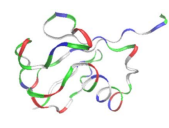

Ribbon
图 $3 \mathrm{VMD}$ 展现的蛋白酶 $\mathrm{CDK} 2^{[10]}$ 分子骨架模型

当前 Cartoon 模型的工作重点在可视化的性能 提升和针对不同分析需求的模型改善. Halm 等 ${ }^{[11]}$ 实现了基于 CPU 的 Cartoon 模型网格细化算法. 近 年来基于 GPU 的渲染方法性能快速提升, 已超过 基于 $\mathrm{CPU}$ 的方法. Hermosilla 等 ${ }^{[12]}$ 使用细化着色器 加快了 Cartoon 模型实时渲染速度. Kocincová 等 ${ }^{[13]}$
提出蛋白质二级结构比较的可视化方法, 通过设 计一维序列图展现蛋白质二级结构信息, 弥补了 传统一维和三维表示分子结构间的缺口. Schulz 等 ${ }^{[14]}$ 提出二级结构不确定性可视化方法, 通过在 Cartoon 模型上设置透明度、模型、频率和振幅等 视觉通道, 帮助用户理解蛋白质二级结构中存在 的不确定性, 这种多视图交互设计使用户能快速 地观察蛋白质间的空间差异, 有效地缓解了三维 可视化中的遮挡问题.

\section{3 分子表面模型可视化}

目前分子表面结构的模型包括范德华表面 (vander Waals surface, vdW)、溶剂可及表面(solventaecessible surface, SAS) ${ }^{[15]}$ 和溶剂排斥表面(solventexcluded surface, SES) ${ }^{[16]}$ 等. 如图 4 所示, $v d W$ 表面 是由每个原子按照范德华半径生成的球面集合, 能清晰地表征分子的空间体积. 通过探针球绕 $\mathrm{vdW}$ 表面滚动时所有球心点组成的表面被称作 SAS 表面, 能够在一定程度上表征溶剂分子与生物 大分子的接触表面，但会引起分子体积膨胀失真; SES 表面由探针球与 vdW 表面接触时的接触面作 为分子表面. 图 5 所示为常见表面模型可视化效果.

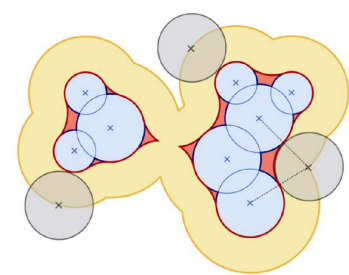

vdW表面; SAS表面; $\quad$ SES表面

图 43 种分子表面结构模型二维示意图 ${ }^{[2]}$
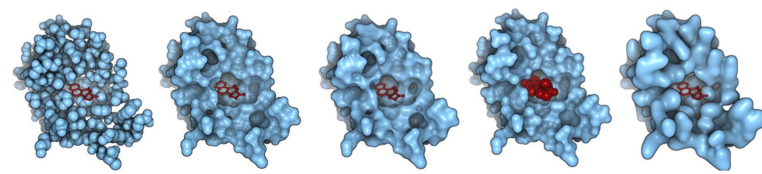

a. vdW

b. SES

c. MSS

d. LES

图 5 蛋白质异构酶(PDB:1OGZ)不同表面可视化模型 ${ }^{[2]}$

SES 表面既能表征溶剂分子与生物分子的接 触情况, 又能表征分子的空间体积, 同时还能辅助 用户感知分子表面的空腔部分, 因而成为分析观 察蛋白质与配体结合位点的重要可视化手段. 在 提升 SES 可视化质量方面, Edelsbrunner ${ }^{[17]}$ 针对 $\mathrm{SES}$ 表面模型的 $C_{1}$ 不连续问题, 提出了图 $5 \mathrm{c}$ 所示 的分子蒙皮表面(molecular skin surface, MSS); 针 对 SES 模型的探针球只能表示单原子溶剂, Lindow 等 ${ }^{[18]}$ 提出了图 $5 \mathrm{~d}$ 所示的表面精度更高的配体排除 
表面(ligand-excluded surface, LES)模型.

图 $5 \mathrm{e}$ 中利用卷积核函数表征原子周围电子密 度，以隐式建模的方式近似分子表面，成为折衷可 视化性能和质量的方案 ${ }^{[19]}$. 通过设计函数参数, 可模拟不同探针球下的溶剂可及表面 ${ }^{[20]}$. Kanamori 等 ${ }^{[21}$ 提出基于核函数的光线投射技术，使用 Bézier 裁剪快速计算光线和曲面的交点. Parulek 等 ${ }^{[22]}$ 设计 了考虑溶剂球半径的隐式函数, 利用隐式空间的 距离等值面近似 SES 表面, 使 Gaussian 方法能更 好地模拟 SES 表面模型.

\section{4 分子空腔模型可视化}

作为生物分子“空”的部分, 空腔经常内嵌或 深埋在分子内部, 人们对空腔的感知, 一直以来都 是生物分子可视化领域的难点.

一种表现空腔的方法是采用光照技术提升分 子表面的景深层次，达到间接感知空腔. 不同的光 照模型对用户感知空腔结构差异性很大. 因此, 设 计合适的光照模型是表现这些空腔结构特征的关 键点之一. 环境光遮蔽 (ambient occlusion, AO) 技 术通过模拟局部空间环境光传播特性形成局部阴 影效果, 能有效地增强物体空间深度感知能力, 非 常适用于如生物大分子此类稠密粒子数据集. 由 于 $A O$ 计算较复杂, 因此研究人员提出一系列针对 $\mathrm{AO}$ 的加速算法, 以实现其交互式可视化. 针对屏 幕空间环境光遮蔽(screen-space ambient occlusion, SSAO)难以应用于空间感知要求更高的生物分子 结构可视化领域, Grottel 等 ${ }^{[23]}$ 提出物理空间环境 光遮蔽(object-space AO, OSAO). OSAO 考虑着色 图元周边某个空间范围所有局部邻接信息, 通过 计算周边环境的阴影体密度来模拟环境光遮蔽效 果. 如图 6 所示, 在分子阴影体处理的基础上, OSAO 能实现由上万原子构成的蛋白质分子快速 可视化. Hermosilla 等 ${ }^{[24]}$ 采用基于范德华球体积估 算且锥体简化光照计算的方法，在提高阴影体计 算速度的前提下，加强了 $\mathrm{AO}$ 的空间感知效果.

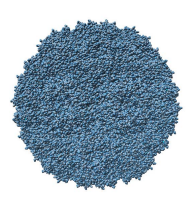

a. Phong

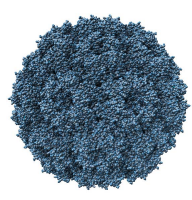

b. SSAO

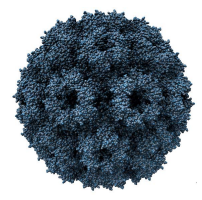

c. OSAO
图 6 不同光照模型下的分子结构可视化效果 ${ }^{[23]}$

更常用的空腔展现方法是将其作为可视化对 象. 空腔计算模型直接影响了空腔可视化效果，且 为更好地理解空腔，常需以分子表面和结构模型
作背景. Krone 等 ${ }^{[25]}$ 采用基于空间网格的计算模型, 采用空腔直接体绘制的方法, 并通过二级结构、截 断面和半透明可视化效果表征分子结构增强空腔 感知. 为更好地在分子表面结构下观察空腔, Lindow 等 ${ }^{[26]}$ 采用骨架形式描述空腔, 并设计镂空及 增加光源的渲染算法加强感知. 可视分析工具 CAVER Analyst ${ }^{[8]}$ 则采用基于 Voronoi 的空腔计算 模型, 其空腔以 Voronoi 顶点为球心、以最远可及 原子范德华球表面为半径的球体集合来描述，并 通过二级结构可视化效果表征分子结构增强空腔 感知.

为弥补三维展现空腔易遮挡、感知困难的缺 陷, 研究人员对空腔非空间可视化进行了研究. 2015 年 Masood 等 ${ }^{[27]}$ 提出图 7 所示的以轴向分别展 现通道长、宽的二维通道轮廓可视化方法 CHEXVIS，用颜色映射描述通过每个位置的宽度、 静电势、亲疏水性等, 设计了内壁氨基酸盒图描述 内壁氨基酸的生化属性. 近年来，面向动态分子结 构的空腔非空间可视化方法多基于此方法, 并针 对其动态性进行了相应的可视化设计.

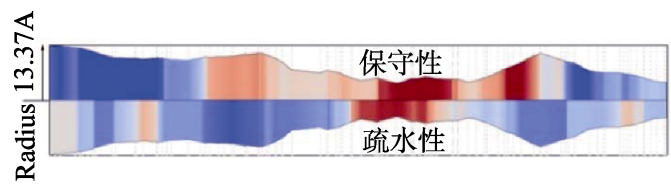

图 7 CHEXVIS 工具可视化分子通道效果 ${ }^{[27]}$

在 3 种分子空间形态可视化基础上，近年来领 域专家从大尺度分析、动态性分析和人机交互等方 面对生物分子可视化技术提出了相应需求, 促进 了可视化技术对大规模生物分子场景可视化、动态 分子结构可视分析和面向虚拟现实(virtual reality, VR)应用的交互式生物分子可视化等的研究.

\section{2 大规模生物分子场景可视化}

介观级生物分子场景的空间范围为 $10 \mathrm{~nm}$ $0.1 \mu \mathrm{m}$, 介于生物分子和细胞尺度之间. 其特点 是: (1) 包含百万级以上原子个数; (2) X 射线、核 磁共振和冷冻电镜等技术无法达到其空间范围的 原子级信息. 尽管介观级分子场景在科学假设、科 研交流、计算模拟与科普中作用重大，其可视化仍 存在无真实数据支撑、数据海量等难题需要解决. 围绕这些关注点, 研究人员从粗粒度生物分子图 像获取分子场景的大致构象出发, 设计自动或半 自动的分子组装技术，以构造整个生物场景，利用 
LOD 技术和基于 GPU 的硬件加速技术来提升场景 的渲染性能, 力图将介观尺度的分子场景展现在 用户眼前.

\section{1 分子组装技术}

目前, 对于介观级生物分子场景, 还无法利 用实验直接获取原子数据. 因此, 根据单分子数 据和分子场景构造规则, 实现自动化或半自动化 分子组装是构造生物分子场景的关键一环. Falk 等 ${ }^{[28]}$ 利用四元数和平移向量将单元分子的移动和 旋转信息存储在 GPU 上, 组装构建出含有微管蛋 白和肌动蛋白分子、共计 176 万个原子的生物分 子场景. Johnson 等 ${ }^{[29]}$ 首次提出了 cellPACK 方法, 基于二维显微镜扫描图像和生物实体的相关知识, 采用基于统计和规则的方法建模，便于快速分子 组装及修改. 为引导对抗病毒传播方面的研究, 用 该方法实现了 HIV 病毒超微结构的原子级组装建 模. Schwarzl 等 ${ }^{[30]}$ 提出了 cellPACKexplorer 系统, 用于帮助发现 cellPACK 分子组装中的错误并改进 组装算法. 该系统专注于可视化 cellPACK 的配置 参数, 通过设计用户界面支持查看基于数值和视 觉 2 种类型的组装效果. Klein 等 ${ }^{[31}$ 提出了基于科 学发现的细胞微管动态多尺度模型, 以生成整个 微管形状及其组合分解的行为动画. 2021 年, Nguyen 等 ${ }^{[32]}$ 利用 cellPACK 技术, 结合生物领域规 则, 首次实现了 SARS-CoV-2 病毒原子级组装建模 及可视化. 如图 8 所示, 人类第 1 次有机会近距离 观察肆虐全球的 SARS-CoV-2 病毒的真面目.

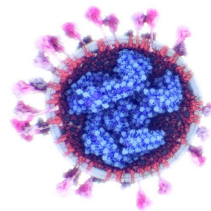

a. 内部绳状结构

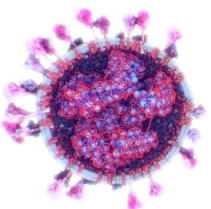

b. RNA 附着

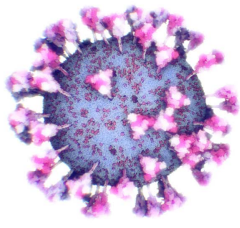

c. 整体外部效果
图 8 SARS-CoV-2 病毒可视化效果 ${ }^{[32]}$

\subsection{LOD 技术}

大规模生物分子场景由百万级以上原子紧密 堆叠, 直接渲染将导致效率低和感知困难, 需从 LOD 人手解决.

蛋白质分子方面的 LOD 设计是针对 $\mathrm{vdW}$ 模型 开展的. Le Muzic 等 ${ }^{[33]}$ 利用顶点着色器控制要生 成的原子数量, 根据不断增加的相机距离调整细 化着色器的镶嵌级别. 通过对原子多级采样, 并缩 放剩余原子的半径, 在 GPU 上动态构建生物分子 的多层次抽象模型. Guo 等 ${ }^{[34]}$ 受 Parulek 等 ${ }^{[22]}$ 利用
层次聚类构建 LOD 提高整体渲染性能的启发, 提 出用于生物分子场景的动态视点相关细节层次的 物体空间误差度量, 在提升渲染性能的同时保证 了可视化精度. 如图 9 所示, 该工作利用生物学方 面的领域知识, 设计了考虑氨基酸残基的分子层 次分类方法，加快了聚类预处理速度.

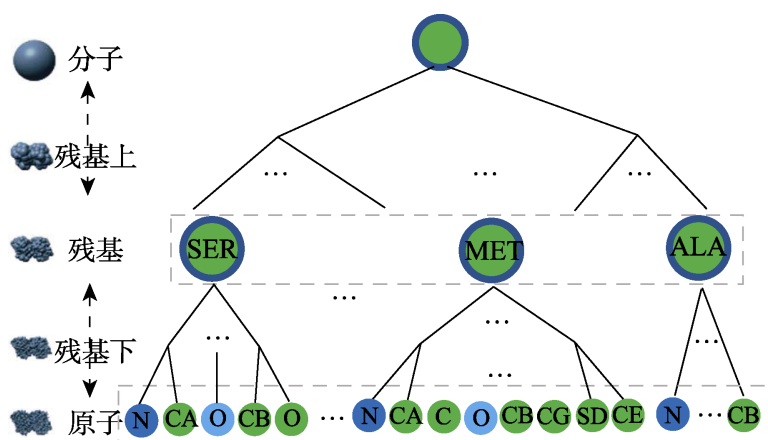

图 9 考虑氨基酸残基的分子层次分类 ${ }^{[34]}$

面向 DNA 的 LOD 技术，通常根据生物学尺度 语义来设定. Miao 等 ${ }^{[35]}$ 提出用语义抽象表示 DNA 纳米 10 个层次结构的方法, 并增加了尺度自适应 交互和平滑过渡, 便于多尺度、直观地修改复杂结 构, 为 DNA 纳米技术的应用提供了多尺度可视化 交互的可能.

2019 年 Miao 等 ${ }^{[36]}$ 综述了多尺度分子可视化 工作，详细阐述并分析了多尺度分子可视化面临 的问题、挑战和任务. Halladjian 等 $^{[37]}$ 提出的 ScaleTrotter，进一步扩展了介观级空间的尺度跨 度, 实现了从细胞核染色体到原子 7 个层次的尺度 二三维可视化设计, 以及各尺度间平滑的视觉切 换. 这些对 SARS-CoV-2 病毒可视化 ${ }^{[32]}$ 起到了技 术支撑作用.

\subsection{GPU 加速技术}

对百万级规模原子组成的稠密生物分子场景, 直接采用基于三角形网格的球体光栅化方法进行 交互性绘制极具挑战性，需借助 GPU 的硬件可编 程和加速优势. 其工作体现在几何图元渲染、遮挡 剔除、光照模型等方面 ${ }^{[36]}$.

在几何图元渲染方面，Tarini 等 ${ }^{[38]}$ 首次采用 Impostor 技术, 输人正方形图元, 在片元着色器上 通过光线投射计算球体形状，结合环境光遮蔽技 术，实现了百万级原子规模的生物分子 $\mathrm{vdW}$ 表面 和球棒结构的交互式渲染. Müller 等 ${ }^{[39]}$ 以点图元为 输人，同样采用光线投射完成 Metaball 渲染，实现 了分子 Gaussian 表面的可视化效果. 目前, 大规模 生物分子场景多采用或参考这 2 种渲染方法 ${ }^{[7,40]}$. 
在稠密球体场景中，遮挡剔除技术能有效地剔 除不必要的图形绘制, 提升渲染效率. Parulek 等 ${ }^{[22]}$ 提出基于 GPU 的“A 缓冲区”技术，通过存储每个 像素中相交原子球离视点远近的链表, 在片元着 色器上动态计算每个像素实际渲染颜色. 与之类 似, Falk 等 ${ }^{[28}$ 提出基于 Impostor 图元的深度剔除算 法. Muzic 等 ${ }^{[40]}$ 提出基于计算着色器的层次 $\mathrm{Z}$ 缓冲 遮挡剔除算法，绘制 6000 万个原子规模的场景性 能较之前提升了近一倍.

\section{3 动态分子结构可视分析技术}

\section{1 分子轨迹可视分析}

分子动力学模拟是对原子和分子的物理运动 及其相互作用的计算模拟方法，被广泛应用于理 论化学、材料科学和生物化学等领域. 聚焦生物分 子的动态特性, 如蛋白质折叠、蛋白质-配体和蛋 白质-蛋白质交互, 能更加准确地了解与分析分子 结构和功能特征. 随着软硬件的进步和新计算模 型的提出, 模拟计算产生的数据量逐渐增加, 如模 拟毫秒级的分子运动，可产生几十万甚至几百万个 时间步数据. 加之分子运动的复杂性, 常见的以统 计功能为主的分析工具, 如 MDAnalysis ${ }^{[51]}$, ProDy ${ }^{[42]}$

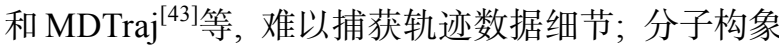

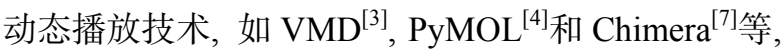
又难以抓住长时间轨迹下的重要环节和事件. 针对 分子动力学轨迹的可视化交互探索技术应运而生.

目前, 分子动力学轨迹的可视化交互探索重 点关注蛋白质-配体、蛋白质-蛋白质分子交互. Furmanová 等 ${ }^{[44]}$ 通过设计概览图和条形图帮助用 户导航到配体轨迹中关注区域, 通过散点图矩阵查 看配体动态行为, 探索不同配体轨迹的特征. 此外, 通过轨迹的自动/交互式简化, 领域专家可使用三 维视图进一步探索配体轨迹空间. 基于 Furmanová 等 ${ }^{[44]}$ 的工作, $\mathrm{Vad}$ 等 ${ }^{[45]}$ 完成了大量水分子轨迹的可 视化探索. Jurčík 等 ${ }^{[46]}$ 设计了图表矩阵满足用户分 析时钻取交互需求，提升了领域专家捕捉感兴趣 信息的效率. David 等 ${ }^{[47]}$ 通过计算并可视化编码信 息引导用户找到配体和蛋白质间发生有趣的相互 作用的区域，采用分层聚类算法提升数据探索的 效率. Byška 等 ${ }^{[48]}$ 提出了基于 Focus+Context 的交 互式视觉分析方法，用于长时间和密集的分子动 力学模拟分析. Furmanová 等 ${ }^{[49]}$ 提出了分子对接轨 迹的可视化分析工具 DockVis, 使用分子对接工具 CaverDock ${ }^{[50]}$ 的输出数据, 将配体及周围环境用
二三维结合的形式描述. 用户利用该工具能快速 感知蛋白质隧道中配体的空间构象, 并与视图交 互开展信息钻取.

\section{2 动态分子空腔可视分析}

空腔动态性分析能够获取动态条件下口袋的 瞬时模式, 如子空腔运动、通道/隧道闭合运动和多 空腔变构运动等 ${ }^{[51]}$, 这些空腔运动直接反映蛋白 质结构的药理性. 其受到生物化学领域的广泛关注, 发展了如 MDPocket ${ }^{[52]}$, CAVER ${ }^{[53]}$, CavityPlus ${ }^{[54]}$ 和 D3Pocket ${ }^{[55]}$ 等多种动态空腔计算分析工具, 有些 已应用于针对 SARS-CoV-2 病毒的药物篎选. 例 如, 中国科学院上海药物研究所开发的 D3Pocket, 已部署在 D3Targets-2019-nCoV 平台下 ${ }^{[56]}$, 可用于 研究药物靶标蛋白上配体结合口袋的动态行为及 相互关系，对参与病毒感染、复制和释放整个过程 的所有潜在靶点蛋白进行药物研发, 并对靶点未 知的活性化合物或有效药物进行靶点蛋白预测.

动态分子结构下, 由于空腔运动复杂且变化 多样, 因此以三维展现、统计图表作为可视化结果 的常规动态空腔计算分析工具, 缺乏对领域用户 交互式探索需求的支持. Krone 等 ${ }^{[25]}$ 提出动态空腔 交互式探索技术，用户可以方便地选定关注的空 腔并跟踪其演变过程; 之后在其扩展子工作 ${ }^{[57]}$ 引 人更多二维图表，如蛋白质序列图、空腔长宽图、 空腔时间-面积折线图和空腔变化关系图，以多角 度地展现动态空腔的特性. Lindow 等 ${ }^{[88]}$ 采用路径 时间线可视化，展现了空腔分裂合并的拓扑事件 随时间的演变. Parulek 等 ${ }^{[59]}$ 用颜色图展现内壁氨 基酸的亲疏水性、极性和电荷属性，用时间散点图 展现氨基酸属性随时间的分布，设计了细节观察 动态空腔的二三维协同可视分析系统.

研究人员针对原动态空腔计算分析需求开发 了相应的可视分析工具. 如图 10 所示, CAVER Analyst ${ }^{[8]}$ 针对 CAVER 空腔计算结果设计了基于长

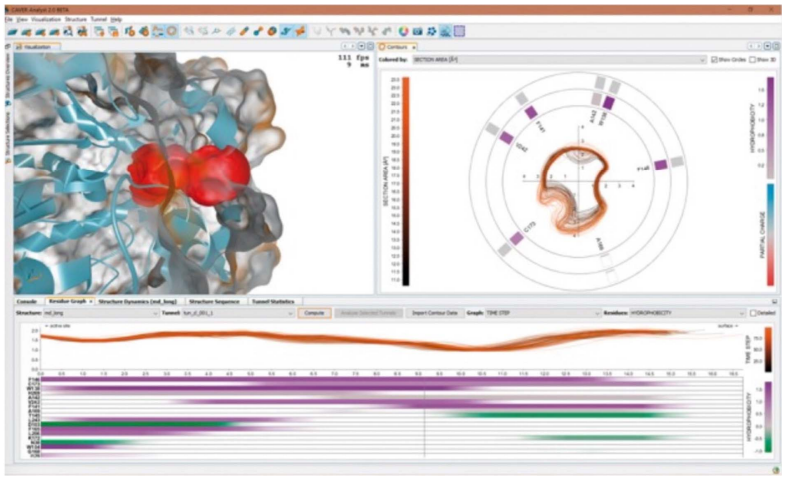

图 10 CAVER Analyst 空腔分析工具 ${ }^{[8]}$ 
宽图的空腔形状与内壁氨基酸关联的可视分析方

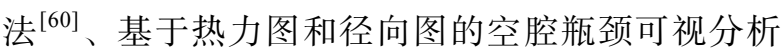
方法 ${ }^{[8]}$. 考虑空腔数据的时空特性, Guo 等 ${ }^{[6]}$ 提出 了时空多尺度空腔可视分析方法, 将空腔在时间 和空间上分为 9 个尺度, 以可视化形式展现每个尺 度的用户关注信息，实现用户多层次、多角度分析 空腔的需求.

\section{4 面向 VR 应用的交互式生物分子可视化}

为更易理解生物大分子间的空间关系，早期 的分子可视化已采用了立体渲染。近年来，VR 显 示和交互设备的出现和发展，更激发了对生物分 子结构和场景的沉浸式可视化与交互式分析的研 究工作.

沉浸式可视化方面，Davison 等 ${ }^{[62]}$ 设计开发了 名为 LifeBrush 的 VR 平台, 用户可在 VR 环境中 绘制动态分子插图，交互式模拟进人该生物分子 结构的效果，可使用户体验中尺度细胞分子场景， 并能从复杂混乱的中尺度环境中交互式地探索驱 动细胞过程中分子间的相互作用; Zhang 等 ${ }^{[63]}$ 设计 开发了 BioVR 交互式 VR 辅助平台, 使用 Unity3D 游戏环境对 DNA, RNA、蛋白质序列和蛋白质结构 进行集成可视分析; Trellet 等 ${ }^{[64]}$ 提出沉浸式环境中 可视化和分析生物分子数据的管线，在领域专家 熟悉的工作环境中对语义链接的二维和三维异构 数据进行直接交互，实现通过描述内容和上下文 语义定义分子场景创建智能系统.

在生物分子场景中, 触觉交互技术允许用户 使用自身的知识和直觉感受分子结构, 并干预其 中. 为解决力稳定性问题，Iakovou 等 ${ }^{[65]}$ 设计了受 力尺度设计、多点碰撞响应和面向对接的触觉导航 的方法，将触觉交互应用于蛋白质分子相互作用 的分析，实现了在生物大分子上进行基于触觉交 互技术刚性对接的虚拟环境; Matthews 等 ${ }^{[66]}$ 提出 面向柔性受体的触觉辅助交互式分子对接方法, 通过显式溶剂分子动力学近似无配体受体分子运 动轨迹来降低计算复杂性, 保证了整个对接过程 的交互性; Iakovou 等 ${ }^{[67]}$ 提出具有实时计算和显示 氢键、多点碰撞检测等功能的 DockIT 工具, 进一 步加深了用户在沉浸式环境中的体验.

\section{5 结 语}

本文系统地介绍了近年来生物大分子可视化
技术的研究工作. 作为人们感知认知微观世界的 界面, 生物分子可视化已经发展多年, 但仍然有以 下方面需要进一步推进.

（1）规模更大、更复杂的生物分子结构测定和 模拟数据将不断出现, 这不仅使对计算资源的需 求日益增加, 也为生物分子可视化带来了挑战. 为 适应这些挑战, 不仅要改进现有分子模型可视化 方法，还需引人新的可视化技术，以显示更大时空 范围的生物分子场景, 实现整个细胞、甚至生物体 在原子细节上的展现. 这需要研究新颖的多尺度 可视化技术，不仅在空间尺度之间，而且在时间尺 度之间建立桥梁，以满足对模拟生物分子系统多 维特性的洞察 ${ }^{[68]}$.

(2) 随着生物化学领域对分子动态特性影响 分子功能的工作不断深化, 如何利用可视化技术分 析不同分子之间以及其子结构之间的关联，设计并 实现定制化可视分析工具, 进而辅助领域专家多角 度感知和关注的生物分子交互特性, 是未来生物分 子可视化与可视分析技术关注的重点之一.

(3) 可视化社区与生物分子社区的密切结合, 关乎可视化技术能否准确把握领域需求, 能否提 升可视化技术影响力. 一种促进社区交叉结合的 有效方法, 是深化可视化社区成果与生物分子领 域已有工具的软件结合, 为专业工具提供插件/组 件服务 ${ }^{[69]}$ ，促进领域用户更多地了解和使用可视

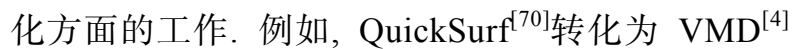
插件, PyMOL ${ }^{[5]}$ 引人了 QuteMol ${ }^{[38]}$ 的可视化工作, CAVER Analyst ${ }^{[8]}$ 为 CAVER $^{[53]}$ 工具提供了空腔可 视分析的平台. 未来的工作将更多地关注与领域 软件的结合, 以体现可视化技术在生物分子领域 的价值.

\section{参考文献(References):}

[1] Burley S K, Berman H M, Charmi B, et al. RCSB Protein Data Bank: biological macromolecular structures enabling research and education in fundamental biology, biomedicine, biotechnology and energy[J]. Nucleic Acids Research, 2019, 47(1): 464-474

[2] Kozlíková B, Krone M, Falk M, et al. Visualization of biomolecular structures: state of the art revisited[J]. Computer Graphics Forum, 2016, 36(8): 178-204

[3] Krone M, Kozlíková B, Lindow N, et al. Visual analysis of biomolecular cavities: state of the art[J]. Computer Graphics Forum, 2016, 35(3): 527-551

[4] Humphrey W, Dalke A, Schulten K. VMD: visual molecular dynamics[J]. Journal of Molecular Graphics, 1996, 14(1): 33-38

[5] Delan W L. PyMOL: an open-source molecular graphics 
tool[J]. CCP4 Newsletter on Protein Crystallography, 2002, 40(1): 82-92

[6] Pettersen E F, Goddard T D, Huang C C, et al. UCSF Chimera-a visualization system for exploratory research and analysis[J]. Journal of Computational Chemistry, 2004, 25(13): 1605-1612

[7] Grottel S, Krone M, Muller C, et al. MegaMol—a prototyping framework for particle-based visualization[J]. IEEE Transactions on Visualization and Computer Graphics, 2015, 21(2): 201-214

[8] Jurck A, Bednar D, Byska J, et al. CAVER Analyst 2.0: analysis and visualization of channels and tunnels in protein structures and molecular dynamics trajectories[J]. Bioinformatics, 2018, 34(20): 3586-3588

[9] Richardson J S. The anatomy and taxonomy of protein structure[J]. Advance in Protein chemistry, 1981, 34(1): 167-339

[10] Kumar S V, Bugg C E, Cook W J. Structure of ubiquitin refined at $1.8 \AA$ resolution[J]. Journal of Molecular Biology, 1987, 194(3): 531-544

[11] Halm A, Offen L, Fellner D W. Visualization of complex molecular ribbon structures at interactive rates[C] //Proceedings of the 8th International Conference on Information Visualization. Los Alamitos: IEEE Computer Society Press, 2004: 737-744

[12] Hermosilla P, Guallar V, Vinacua A, et al. Instant visualization of secondary structures of molecular models[C] //Proceedings of the Eurographics Workshop on Visual Computing for Biology and Medicine. Aire-la-Ville: Eurographics Association Press, 2015: 51-60

[13] Kocincová L, Jarešová M, Byška J, et al. Comparative visualization of protein secondary structures[J]. BMC Bioinformatics, 2017, 18(2): 1-12

[14] Schulz C, Schatz K, Krone M, et al. Uncertainty visualization for secondary structures of proteins[C] //Proceedings of the IEEE Pacific Visualization Symposium. Los Alamitos: IEEE Computer Society Press, 2018: 96-105

[15] Lee B, Richards F M. The interpretation of protein structures: estimation of static accessibility[J]. Journal of Molecular Biology, 1971, 55(3): 379-380

[16] Richards F M. Areas, volumes, packing and protein structure[J]. Annual Review of Biophysics and Bioengineering, 1977, 6(6): 151-176

[17] Edelsbrunner H. Deformable smooth surface design[J]. Discrete \& Computational Geometry, 1999, 21(1): 87-115

[18] Lindow N, Baum D, Hege H C. Ligand excluded surface: a new type of molecular surface[J]. IEEE Transactions on Visualization and Computer Graphics, 2014, 20(12): 2486-2495

[19] Blinn J F. A Generalization of algebraic surface drawing[J]. ACM Transactions on Graphics, 1982, 1(3): 235-256

[20] Grant J A, Pickup B T. A Gaussian description of molecular shape[J]. Journal of Physical Chemistry, 1995, 99(11): 3503-3510

[21] Kanamori Y, Szego Z, Nishita T. GPU-based fast ray casting for a large number of metaballs[J]. Computer Graphics Forum, 2008, 27(2): 351-360

[22] Parulek J, Jönsson D, Ropinski T, et al. Continuous levels-of-detail and visual abstraction for seamless molecular visualization[J]. Computer Graphics Forum, 2014, 33(6): 276-287
[23] Grottel S, Krone M, Scharnowski K, et al. Object-space ambient occlusion for molecular dynamics[C] //Proceedings of the IEEE Pacific Visualization Symposium. Los Alamitos: IEEE Computer Society Press, 2012: 209-216

[24] Hermosilla P, Guallar V, Vinacua A, et al. High quality illustrative effects for molecular rendering $[\mathrm{J}]$. Computers \& Graphics, 2015, 54(1): 113-120

[25] Krone M, Falk M, Rehm S, et al. Interactive exploration of protein cavities[J]. Computer Graphics Forum, 2011, 30(3): 673-682

[26] Lindow N, Baum D, Hege H C, et al. Voronoi-based extraction and visualization of molecular paths[J]. IEEE Transactions on Visualization and Computer Graphics, 2011, 17(12): 2025-2034

[27] Masood T B, Sandhya S, Chandra N, et al. CHEXVIS: a tool for molecular channel extraction and visualization[J]. BMC Bioinformatics, 2015, 16: Article No.119

[28] Falk M, Krone M, Ertl T. Atomistic visualization of mesoscopic whole-cell simulations using ray-casted instancing[J]. Computer Graphics Forum, 2013, 32(8): 195-206

[29] Johnson G T, Autin L, Al-Alusi M, et al. cellPACK: a virtual mesoscope to model and visualize structural systems biology[J]. Nature Methods, 2015, 12(1): 85-91

[30] Schwarzl M, Autin L, Johnson G, et al. cellPACKexplorer: interactive model building for volumetric data of complex cells[J]. Computers \& Graphics: X, 2019, 2: 100010

[31] Klein T, Viola I, Groeller E, et al. Multi-scale procedural animations of microtubule dynamics based on measured data[J]. IEEE Transactions on Visualization and Computer Graphics, 2020, 26(1): 622-632

[32] Nguyen N, Strnad O, Klein T, et al. Modeling in the time of COVID-19: statistical and rule-based mesoscale models[J]. IEEE Transactions on Visualization and Computer Graphics, 2021, 27(2): 722-732

[33] Le Muzic M, Parulek J, Stavrum A K, et al. Illustrative visualization of molecular reactions using omniscient intelligence and passive Agents[J]. Computer Graphics Forum, 2014, 33(3): 141-150

[34] Guo D L, Nie J L, Liang M, et al. View-dependent level-of-detail abstraction for interactive atomistic visualization of biological structures[J]. Computers \& Graphics, 2015, 52: $62-71$

[35] Miao H, Llano E D, Sorger J, et al. Multiscale visualization and scale-adaptive modification of DNA nanostructures[J]. IEEE Transactions on Visualization and Computer Graphics, 2017, 24(1): 1014-1024

[36] Miao H, Klein T, Kouřil D, et al. Multiscale molecular visualization[J]. Journal of Molecular Biology, 2019, 431(6): 1049-1070

[37] Halladjian S, Miao H, Kouil D, et al. ScaleTrotter: illustrative visual travels across negative scales[J]. IEEE Transactions on Visualization and Computer Graphics, 2020, 26(1): 654-664

[38] Tarini M, Cignoni P, Montani C. Ambient occlusion and edge cueing for enhancing real time molecular visualization[J]. IEEE Transactions on Visualization \& Computer Graphics, 2006, 12(5): $1237-1244$

[39] Müller C, Grottel S, Ertl T. Image-space GPU metaballs for time-dependent particle data sets $[\mathrm{C}] / /$ Proceedings of the $\mathrm{Vi}$ - 
sion, Modeling, and Visualization Conference. New York: ACM Press, 2007: 1-10

[40] Muzic M L, Autin L, Parulek J, et al. cellVIEW: a Tool for illustrative and multi-scale rendering of large biomolecular datasets[C] //Proceedings of the Eurographics Workshop on Visual Computing for Biomedicine. Aire-la-Ville: Eurographics Association Press, 2015: 61-70

[41] Agrawal N M, Denning E J, Woolf T B, et al. MDAnalysis: a toolkit for the analysis of molecular dynamics simulations[J]. Journal of Computational Chemistry, 2011, 32(10): 2319-2327

[42] Bakan A, Meireles L M, Bahar I. ProDy: protein dynamics inferred from theory and experiments[J]. Bioinformatics, 2011, 27(11): 1575-1577

[43] Mcgibbon R T, Beauchamp K A, Schwantes C R, et al. MDTraj: a modern open library for the analysis of molecular dynamics trajectories[J]. Biophysical Journal, 2014, 109(8): 1528-1532

[44] Furmanová K, Jareová M, Byka J, et al. Interactive exploration of ligand transportation through protein tunnels[J]. BMC Bioinformatics, 2016, 18(Suppl 2): Article No.22

[45] Vad V, Byška J, Jurcík A, et al. Watergate: visual exploration of water trajectories in protein dynamics[C] //Proceedings of Eurographics Workshop on Visual Computing for Biology and Medicine. Aire-la-Ville: Eurographics Association Press, 2017: $33-42$

[46] Jurčík A, Furmanová K, Byška J, et al. Visual analysis of ligand trajectories in molecular dynamics[C] //Proceedings of the IEEE Pacific Visualization Symposium. Los Alamitos: IEEE Computer Society Press, 2019: 212-221

[47] David D, Pedro H, Timo R, et al. Visualization of large molecular trajectories[J]. IEEE Transactions on Visualization and Computer Graphics, 2019, 25(1): 987-996

[48] Byška J, Trautner T, Marques S M, et al. Analysis of long molecular dynamics simulations using interactive focus + context visualization[J]. Computer Graphics Forum, 2019, 38(3): 441-453

[49] Furmanová K, Vávra O, Kozlíková B. DockVis: visual analysis of molecular docking trajectories[J]. Computer Graphics Forum, 2020, 39(6): 452-464

[50] Vavra O, Filipovic J, Plhak J, et al. CaverDock: a molecular docking-based tool to analyse ligand transport through protein tunnels and channels[J]. Bioinformatics, 2019, 35(23): 4986-4993

[51] Stank A, Kokh D B, Fuller J C, et al. Protein binding pocket dynamics[J]. Accounts of Chemical Research, 2016, 49(5): 809-815

[52] Barril X. MDPocket: open-source cavity detection and characterization on molecular dynamics trajectories[J]. Bioinformatics, 2011, 27(23): 3276-3285

[53] Chovancova E, Pavelka A, Benes P, et al. CAVER 3.0: a tool for the analysis of transport pathways in dynamic protein structures[J]. PLoS Computational Biology, 2012, 8(10): e10027081

[54] Xu Y J, Wang S W, Hu Q W, et al. CavityPlus: a web server for protein cavity detection with pharmacophore modelling, allosteric site identification and covalent ligand binding ability prediction[J]. Nucleic Acids Research, 2018, 46(1): 374-379

[55] Chen Z Q, Zhang X B, Peng C, et al. D3Pockets: a method and web server for systematic analysis of protein pocket dynamics[J]. Journal of Chemical Information and Modeling, 2019, 59(8): 3353-3358

[56] Shi Y L, Zhang X B, Mu K J, et al. D3Targets-2019-nCoV: a webserver for predicting drug targets and for multi-target and multi-site based virtual screening against COVID-19[J]. Acta Pharmaceutica Sinica B, 2020, 10(7): 1239-1248

[57] Krone M, Kauker D, Reina G, et al. Visual analysis of dynamic protein cavities and binding sites[C]//Proceedings of the IEEE Pacific Visualization Symposium. Los Alamitos: IEEE Computer Society Press, 2014: 301-305

[58] Lindow N, Baum D, Bondar A N, et al. Exploring cavity dynamics in biomolecular systems[J]. BMC Bioinformatics, 2013, 14: Article No.S5

[59] Parulek J, Turkay C, Reuter N, et al. Visual cavity analysis in molecular simulations[J]. BMC Bioinformatics, 2013, 14: Article No.S4

[60] Byska J, Muzic M L, Groller M E, et al. AnimoAminoMiner: exploration of protein tunnels and their properties in molecular dynamics[J]. IEEE Transactions on Visualization and Computer Graphics, 2016, 22(1): 747-756

[61] Guo D L, Han D X, Xu X M, et al. Spatiotemporal multiscale molecular cavity visualization and visual analysis[J]. Journal of Visualization, 2020, 23: 661-676

[62] Davison T, Samavati F, Jacob C. LifeBrush: painting interactive agent-based simulations[J]. Computers \& Graphics, 2019, 82(1): 232-242

[63] Zhang J F, Paciorkowski A R, Craig P A, et al. BioVR: a platform for virtual reality assisted biological data integration and visualization[J]. BMC Bioinformatics, 2019, 20: Atticle No. 78

[64] Trellet M, Férey N, Flotyński J, et al. Semantics for an integrative and immersive pipeline combining visualization and analysis of molecular data[J]. Journal of Integrative Bioinformatics, 2018, 15(2): 20180004

[65] Iakovou G, Hayward S, Laycock S D. Virtual environment for studying the docking interactions of rigid biomolecules with haptics $[\mathrm{J}]$. Journal of Chemical Information and Modeling, 2017, 57(5): 1142-1152

[66] Matthews N, Kitao A, Laycock S, et al. Haptic-assisted interactive molecular docking incorporating receptor flexibility[J]. Journal of Chemical Information and Modeling, 2019, 59(6): 2900-2912

[67] Iakovou G, Alhazzazi M, Hayward S, et al. DockIT: a tool for interactive molecular docking and molecular complex construction[J]. Bioinformatics, 2020, 36(24): 5698-5700

[68] Schatz K, Krone M, Pleiss J, et al. Interactive visualization of biomolecules' dynamic and complex properties: state of the art and project review $[\mathrm{J}]$. The European Physical Journal Special Topics, 2019, 227: 1725-1739

[69] Alharbi N, Alharbi M, Martinez X, et al. Molecular visualization of computational biology data: a survey of surveys[C] //Proceedings of Eurographics Conference on Visualization. Aire-la-Ville: Eurographics Association Press, 2017: 133-137

[70] Krone M, Stone J E, Ertl T, et al. Fast visualization of Gaussian density surfaces for molecular dynamics and particle system trajectories[C] //Proceedings of Eurographics Conference on Visualization. Aire-la-Ville: Eurographics Association Press, 2012: $67-71$ 